\title{
Idiopathic Hypereosinophilic Syndrome with Multiple Organ Involvement
}

\author{
Reham Abo Shdid $^{a}$ Bahjat Azrieh ${ }^{a}$ Seham Alebbi ${ }^{a}$ Shafik Mansour ${ }^{a}$ \\ Muhammad Naeem ${ }^{b}$ \\ aDepartment of Medical Education, Hamad Medical Corporation, Doha, Qatar; ${ }^{b}$ Hamad \\ General Hospital, Hamad Medical Corporation, Doha, Qatar
}

\begin{abstract}
Keywords
Hypereosinophilic syndrome · Eosinophilia - Idiopathic hypereosinophilic syndrome ·

Multiple organ involvement
\end{abstract}

\begin{abstract}
Idiopathic hypereosinophilic syndrome is a rare disease which is diagnosed after excluding other conditions. The syndrome is characterized by multiple organ involvement including the heart, nervous system, lungs, and gastrointestinal tract. The disease is suspected if there is peripheral blood eosinophilia and no clear etiology. The main treatment is corticosteroids. Patients who do not respond to corticosteroids can be treated with imatinib, immunomodulatory agents, myelosuppressive therapy, or mepolizumab. Alemtuzumab can be considered in severe cases that are unresponsive to other therapies. In this paper, we describe a case of idiopathic hypereosinophilic syndrome with mainly cardiac system involvement and left ventricular thrombus formation which was complicated by cerebral thromboemboli while on warfarin with international normalized ratio in the therapeutic range. Our patient responded well to steroids appreciated by improvement in clinical symptoms and decrease in eosinophil count.

\section{Introduction}

Hypereosinophilic syndrome (HES) is a group of disorders marked by the sustained overproduction of eosinophils in which eosinophilic infiltration and mediator release cause damage to multiple organs. HES is further subclassified, according to the pathogenic mecha- 
nisms resulting in eosinophil expansion, into primary, secondary, or idiopathic. In idiopathic hypereosinophilic syndrome (IHES), the underlying cause of hypereosinophilia remains unknown despite thorough etiologic workup.

Eosinophilic myocarditis is a major cause of morbidity and mortality among patients with HES. It is characterized by myocardial infiltration with eosinophils and lymphocytes followed by myocardial necrosis. Common cardiac presentations include heart failure, chest pain, arrhythmia, and cardiac thrombi. In this paper, we describe a case of idiopathic HES with cardiac, pulmonary, and central nervous system involvement.

\section{Case Presentation}

A 65-year-old African gentleman who was known to have asthma and who recently came from his home country presented with a 2-month history of central chest pain and epigastric pain associated with shortness of breath and nausea. The pain was intermittent, not related to exertion, lasted 10-15 min each time, and improved when leaning forward. Shortness of breath was present on exertion as well as at rest. He denied any fever or cough. He had constipation for several months.

Physical exam was significant for decreased breath sounds in the base of both lungs and epigastric tenderness. Investigations revealed:

- Leukocytosis $\left(19.3 \times 10^{9} / \mathrm{L}\right)$ with eosinophil cell count $7.9 \times 10^{9} / \mathrm{L}$ ( $42 \%$ of leukocytes) and normocytic normochromic anemia but no thrombocytopenia. Hypersensitive C-reactive protein level was $72 \mathrm{mg} / \mathrm{L}$ (normally $0-5 \mathrm{mg} / \mathrm{L}$ ).

- Leukocytosis $\left(19.3 \times 10^{9} / \mathrm{L}\right)$ with eosinophil cell count $7.9 \times 10^{9} / \mathrm{L}$ (42\% of leukocytes) and normocytic normochromic anemia but no thrombocytopenia. Hypersensitive C-reactive protein level was $72 \mathrm{mg} / \mathrm{L}$ (normally 0-5 mg/L).High-sensitive troponin T was elevated (340 ng/L). Creatine kinase MB was normal.

- Leukocytosis $\left(19.3 \times 10^{9} / \mathrm{L}\right)$ with eosinophil cell count $7.9 \times 10^{9} / \mathrm{L}(42 \%$ of leukocytes) and normocytic normochromic anemia but no thrombocytopenia. Hypersensitive C-reactive protein level was $72 \mathrm{mg} / \mathrm{L}$ (normally $0-5 \mathrm{mg} / \mathrm{L}$ ).High-sensitive troponin T was elevated (340 ng/L). Creatine kinase MB was normal.proBNP was elevated $(4,741 \mathrm{pg} / \mathrm{mL}$ ).

Renal function was normal. Urinalysis was normal. Anemia workup showed that iron indices were low. Serum vitamin B12 level was normal. Folic acid level was low. Thyroid function was normal. Fecal occult blood test was negative.

Chest X-ray showed increased cardiothoracic ratio, pulmonary congestion, and bilateral pleural effusion more on the right side. Electrocardiography revealed sinus tachycardia. Echocardiogram showed 4-chamber dilation with global left ventricular hypokinesia and a large, layered, apical thrombus. Ejection fraction was 27\%. Cardiac magnetic resonance imaging (MRI) showed global left ventricular hypokinesia; apical, left ventricular thrombus; and endomyocardial fibrosis.

The patient was admitted as a case of heart failure presumably due to myocarditis. He was not known to have coronary artery disease, and coronary angiography was done 2 months earlier which showed normal coronary arteries.

He was treated with antibiotics and heart failure medications. Warfarin was started for anticoagulation for left ventricular thrombus along with bridging with enoxaparin.

Unfortunately, 2 days later, the patient had left arm and leg weakness. Computed tomography of the head without contrast showed no intracranial hemorrhage but multiple scattered subcortical white matter hypodense foci bilaterally in the centrum semiovale, left frontal, and right temporal regions that were likely ischemic/embolic in origin. We suspected HES and started to search for possible causes.

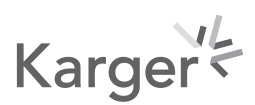


Serum immunoglobulin E level was 4,377 IU/mL (normally 0-114 IU/mL). Serum tryptase level was normal. Screening for anti-nuclear antibodies and anti-neutrophil cytoplasmic antibodies was negative. Serologic tests were negative for viruses (COVID-19 PCR, Epstein-Barr virus, cytomegalovirus, hepatitis B and C viruses, HIV). Screening for parasites was negative. Schistosoma serology was negative. Blood and urine cultures were sterile.

Peripheral blood smear revealed mild normocytic normochromic anemia, neutrophilic leukocytosis with eosinophilia, mild basophilia, and normal platelets. Abdominal ultrasound showed no hepatomegaly or splenomegaly. Bone marrow biopsy showed trilineage hematopoiesis with an increase in eosinophils and their precursors but no increase in blasts.

FISH analysis for FIP1L1-PDGFRA (Fip1-like 1-platelet-derived growth factor receptor alpha), PDGFRB (platelet-derived growth factor receptor beta), and BCR/ABL was negative. Molecular analysis for V617F missense mutation within JAK-2 (Janus Kinase-2) gene was negative. Flow cytometry of peripheral blood was normal. PET scan showed no significant findings except for mildly increased uptake in some segments of the rectum.

A multi-disciplinary team meeting was done involving cardiology, hematology, and internal medicine specialties. After full discussion of the case, the diagnosis of idiopathic HES was carried out and the patient was started on oral prednisolone $60 \mathrm{mg}$ daily with eosinophil count returning to normal on the next day and clinical improvement markedly achieved a few days after.

Regarding his chronic epigastric pain, esophagogastroduodenoscopy was done in another hospital 2 months prior to this admission and showed gastritis. Helicobacter pylori testing was negative. Unfortunately, no more data was available to confirm the eosinophilic gastritis.

Sigmoidoscopy was done during this admission and showed 3 polyps reported by pathology as tubular adenomas. No other pathologies were found.

The patient's epigastric pain and dyspnea clinically significantly improved a few days after starting prednisolone and he was discharged to a rehabilitation facility to continue physical therapy for left arm and leg weakness.

\section{Discussion}

HES is characterized by the presence of marked unexplained blood and tissue eosinophilia associated with a variety of clinical manifestations. Since 1975, 3 criteria have been used to define HES:

(1) blood eosinophilia $>1,500 / \mathrm{mm}^{3}$ for longer than 6 months (or death before 6 months associated with signs and symptoms of hypereosinophilic disease),

(2) lack of evidence for parasitic, allergic, or other known causes of eosinophilia, and

(3) presumptive signs of organ involvement, such as heart failure, gastrointestinal dysfunction, central nervous system abnormalities, fever, or weight loss [1].

However, as it is unlikely that a patient with symptomatic HES would remain untreated for 6 months given the availability of effective therapies that can reduce eosinophilia before irreversible damage occurs, the currently accepted definition of HES is [2]:

(1) Blood eosinophilia of greater than $1,500 / \mathrm{mm}^{3}$ on at least 2 occasions or evidence of prominent tissue eosinophilia associated with symptoms and marked blood eosinophilia.

(2) Exclusion of secondary causes of eosinophilia, such as parasitic or viral infections, allergic diseases, drug-induced or chemical-induced eosinophilia, hypoadrenalism, and neoplasms.

There are 6 clinical variants of HES: myeloproliferative HES, lymphocytic variant HES, overlap HES, associated HES, familial HES, and idiopathic HES [3]. According to the latest criteria of HES, with exclusion of primary and secondary causes of eosinophilia, our case can

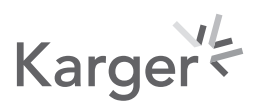


be diagnosed as idiopathic HES. But our case involved a patient with a left ventricular thrombus and asthma, which may or may not be related to HES.

Although there are controversies about how to define eosinophilic end organ damage, evidence of eosinophil-mediated tissue damage using methods other than histology is accepted with highly specific abnormalities that include thrombosis documented by imaging studies as proved in our case [4].

The clinical manifestations of HES are variable.

\section{Cardiac Manifestation}

Cardiac manifestation is the major cause of morbidity in patients with HES and it is found in more than $50 \%$ of patients with either right or left ventricular, pericardial, or global myocardial manifestation. Clinical features range from heart failure to arterial embolism, and they are due to thickening of the endocardium and mural left ventricular thrombosis.

Although cardiac involvement is best diagnosed by means of endomyocardial biopsy, this technique can have a high morbidity and mortality and be difficult to perform. Instead, modern MRI and echocardiography are able to detect fibrosis, eosinophilic infiltrates, and thrombi to stage the fibrotic evolution of the disease [5].

Eosinophil-mediated heart damage evolves through 3 stages, although these stages may be overlapping and not clearly sequential [6]:

- $\quad$ an acute necrotic stage;

- an acute necrotic stage;an intermediate phase characterized by thrombus formation along the damaged endocardium; and

- an acute necrotic stage;an intermediate phase characterized by thrombus formation along the damaged endocardium; anda fibrotic stage characterized by altered cardiac function/heart failure due to either restrictive cardiomyopathy and/or compromise/ entrapment of the chordae tendineae leading to mitral and tricuspid regurgitation.

According to the stages mentioned above, our case fits stage 3 as the patient presented clinically with features of heart failure and was found to have moderate mitral regurgitation, cardiomegaly, restrictive cardiomyopathy, with evidence of intracardiac thrombi on echocardiography and cardiac MRI.

\section{Neurologic Disease}

HES may be complicated by cerebral thromboemboli, encephalopathy, peripheral neuropathy, or longitudinal and/or transverse sinus thrombosis [7]. Cerebral thromboemboli can arise from intracardiac thrombi and manifest as embolic strokes or transient ischemic episodes. MRI can reveal multiple infarcts in watershed areas [8], as seen in our case.

\section{Pulmonary Involvement}

Pulmonary involvement is common in HES and may result from eosinophilic infiltration of the lung with subsequent fibrosis, heart failure, or pulmonary emboli [9]. Abnormal findings seen on imaging include parenchymal infiltrates (37\%), pleural effusion (14\%), intrathoracic lymphadenopathy (12\%), and pulmonary emboli (4\%) [6]. All these findings were seen in our case except for pulmonary emboli.

Other comorbidities, such as asthma, can mask the accurate diagnosis of HES with pulmonary involvement. Chrug-Strauss syndrome should be suspected in any patient with asthma when there is at least 1 of 3 characteristics: difficult to treat, steroid-dependent, and late-onset disease [10], which have all been excluded in our case. In addition, although ChurgStrauss syndrome may involve similar organs to those affected by HES, the absence of renal involvement, vasculitis, and the presence of bone marrow findings in our patient helped to distinguish HES from it.

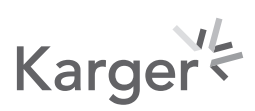




\section{Gastrointestinal Disorders}

Eosinophilic gastritis, enteritis, and/or colitis may occur secondary to HES and cause weight loss, abdominal pain, vomiting, and/or severe diarrhea [9]. Our patient had evidence of gastritis on esophagogastroduodenoscopy.

The differential diagnosis of hypereosinophilia includes malignant disease, parasitosis, and other infections. All of them have been ruled out in our case and the diagnosis of idiopathic HES has been made.

\section{Management of Idiopathic HES}

Patients with idiopathic HES should be treated in the first instance with corticosteroids [11]. Patients requiring emergency treatment for severe or life-threatening eosinophilia, as in our case, should receive high-dose corticosteroids (Grade 1B) [11].

Patients receiving corticosteroids, in whom there is a risk of Strongyloides infection, should receive concomitant Ivermectin to prevent potentially fatal hyperinfection syndrome (Grade 1B) [11]. Although our patient's stool samples were negative for parasites, we decided to give him ivermectin for 2 days because he came from a highly endemic area. Our patient responded well to steroids and eosinophil count returned to normal with no subsequent complications.

Patients with idiopathic HES who do not respond adequately to corticosteroids, who require prolonged corticosteroid therapy, or who are intolerant of corticosteroids should be considered for a short trial (4-6 weeks) of imatinib, immunomodulatory agents (interferon alpha, ciclosporin, or azathioprine), myelosuppressive therapy (hydroxycarbamide), or monoclonal antibody therapy with mepolizumab (anti-interleukin 5), the latter preferably as part of a clinical trial (Grade 2B) [11].

Alemtuzumab, an anti-CD52 monoclonal antibody, should be considered for patients with severe idiopathic HES unresponsive to other therapies, and may be useful in patients with idiopathic HES-associated cardiac and cerebral dysfunction (Grade 2B) [11].

\section{Management of Thrombotic Complications of HES}

The limited literature suggests that perhaps a quarter of HES patients develop thromboembolic complications and that $5-10 \%$ die of them. There is also the suggestion that thromboembolic disease associated with HES is particularly difficult to control [12].

In the complete absence of information about whether anticoagulation could prevent the progression of HES cardiac disease from the thrombotic to the fibrotic stage, prophylactic treatment for this indication cannot be recommended. However, HES patients with evidence of intracardiac thrombosis by echocardiography or cardiac MRI should be treated with anticoagulant therapy because of reports, limited though they are, that $\sim 25 \%$ of these patients develop emboli $[12,13]$.

Warfarin is usually initiated for a mural thrombus in the heart or venous thrombus in an intracranial sinus. However, there is no consensus on the target international normalized ratio. Warfarin should be begun simultaneously with heparin or soon thereafter. Heparin must be continued for at least 5 days and until warfarin has achieved a stable international normalized ratio between 2 and 3 . The transition from heparin to warfarin should not be hurried since unstable anticoagulation at this early stage risks treatment failure [12].

The recommended duration of anticoagulation is based upon the assumption that the risk of recurrent thrombosis and/or embolism is high until or unless the inciting cause(s) is eliminated. Therefore, in patients with HES, the duration of anticoagulation should be determined by the activity of the patient's endomyocardial disease. If this is controlled and if echocardiography reveals the absence of thrombus or resolution of thrombus, it is reasonable to discontinue anticoagulation. The balance between the risks and benefits of anticoagulation, however, must be continuously reassessed [12].

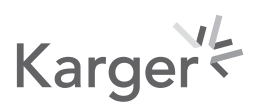




\section{Conclusion}

HES can affect multiple organs. Full investigations are needed to eliminate other causes of hypereosinophilia. In our case, we saw a wide range of systems involvement. Initiation of corticosteroids is essential and emergent, specially if the patient presents with severe or lifethreatening organ failure. Some cases are resistant to corticosteroids and alternative therapies may be needed. Lastly, the thromboembolic disease associated with HES is particularly difficult to control.

\section{Acknowledgement}

We thank Qatar National Library for funding this article and we thank the internal medicine residency program research group at Hamad Medical Corporation.

\section{Statement of Ethics}

This article was approved by Hamad Medical Corporation Medical Research Center. This research was conducted ethically in accordance with the World Medical Association Declaration of Helsinki. The subject in this paper has given his informed consent to publish his case.

\section{Conflict of Interest Statement}

The authors have nothing to disclose.

\section{Funding Sources}

This article was funded by Qatar National Library.

\section{Authors Contributions}

Reham Abo Shdid: writing the manuscript; Bahjat Azrieh: clinical management; Seham Alebbi: clinical management; Shafik Mansour: clinical management; Muhammad Naeem: writing and editing.

\section{References}

1 Chusid MJ, Dale DC, West BC, Wolff SM. The hypereosinophilic syndrome: analysis of fourteen cases with review of the litarature. Medicine. 1975;54(1):1-27. https://europepmc.org/article/med/1090795.

2 Simon HU, Rothenberg ME, Bochner BS, Weller PF, Wardlaw AJ, Wechsler ME, et al. Refining the definition of hypereosinophilic syndrome. J Allergy Clin Immunol. 2010;126(1):45-9.

3 Klion AD. Eosinophilia: a pragmatic approach to diagnosis and treatment. Hematology Am Soc Hematol Educ Program. 2015;2015:92-7.

4 Valent P, Klion AD, Horny HP, Roufosse F, Gotlib J, Weller PF, et al. Contemporary consensus proposal on criteria and classification of eosinophilic disorders and related syndromes. J Allergy Clin Immunol. 2012; 130(3):607-612.e9.

5 Kleinfeldt T, Nienaber CA, Kische S, Akin I, Turan RG, Körber T, et al. Cardiac manifestation of the hypereosinophilic syndrome: new insights. Clin Res Cardiol. 2010;99(7):419-27.

\section{Karger's}


6 Roufosse F, Klion AD, Weller PF. Hypereosinophilic syndromes: Clinical manifestations, pathophysiology, and diagnosis. UpToDate. [cited 2020 Jul 4]. Available from: https://www.uptodate.com/contents/hypereosinophilic-syndromes-clinical-manifestations-pathophysiology-and-diagnosis

7 Moore PM, Harley JB, Fauci AS. Neurologic dysfunction in the idiopathic hypereosinophilic syndrome. Ann Intern Med. 1985;102(1):109-14.

8 Aida L, Parkhutik V, Tembl JI, Martín N, Frasquet M, Bataller L. Embolism and impaired washout: a possible explanation of border zone strokes in hypereosinophilic syndrome. J Neurol Sci. 2013;325(1-2):162-4.

9 Roufosse FE, Goldman M, Cogan E. Hypereosinophilic syndromes. Orphanet J Rare Dis. 2007;2:37.

10 Hayashi S, Furuya S, Imamura H. Fulminant eosinophilic endomyocarditis in an asthmatic patient treated with pranlukast after corticosteroid withdrawal. Heart. 2001;86(3):E7.

11 Butt NM, Lambert J, Ali S, Beer PA, Cross NC, Duncombe A, et al. Guideline for the investigation and management of eosinophilia. Br J Haematol. 2017;176(4):553-72.

12 Ogbogu PU, Rosing DR, Horne MK. Cardiovascular manifestations of hypereosinophilic syndromes. Immunol Allergy Clin North Am. 2007;27(3):457-75.

13 Spry CJ, Davies J, Tai PC, Olsen EG, Oakley CM, Goodwin JF. Clinical features of fifteen patients with the hypereosinophilic syndrome. Q J Med. 1983;52(205):1-22. 